\title{
Hall basis of twisted Lie algebras
}

\author{
Marc Aubry
}

Received: 8 June 2009 / Accepted: 5 January 2010 / Published online: 6 February 2010

(C) Springer Science+Business Media, LLC 2010

\begin{abstract}
In this paper we define a minimal generating system for the free twisted Lie algebra. This gives a correct formulation and a proof to an old statement of Barratt. To this aim we use properties of the Lyndon words and of the Klyachko idempotent which generalize to twisted Hopf algebras some similar results well known in the classical case.
\end{abstract}

Keywords Twisted Hopf algebras · Twisted Lie algebras · Klyachko idempotent · Hall basis · Dynkin word

\section{Introduction}

We can date the birth of twisted algebraic structures from the article of Barratt [2], where he proposed a new way for tackling the study of James-Hopf and HiltonHopf invariants. Many years later general combinatorial foundations of twisted algebraic structures were developed: elementary, combinatorial definitions by Stover [12] copied from the classical (nontwisted) ones; abstract, categorical definitions with the species of structures $[6,10]$. Let us also mention an operadic approach $[3,7,8]$.

The results presented hereafter were announced in [1].

At the end of [2], Barratt gives a description of the linear basis of the free twisted Lie algebra, but without proof. Briefly, he asserts that the free twisted Lie algebra on a set of variables $X$ is generated as a twisted module by the Lyndon words (in the classical meaning) and the brackets $[\ldots[x, x] \ldots, x], x \in X$.

This set is minimal, but it is not generating. To get a feeling of what happens, we consider the following analogy. Like free twisted Lie algebras, free graded Lie

M. Aubry $(\bowtie)$

Laboratoire de Mathématiques Jean-Alexandre Dieudonné UMR CNRS n ${ }^{0}$ 6621, Université de Nice, Parc Valrose, 06108 Nice cedex 02, France

e-mail: aubry@math.unice.fr 
algebras satisfy $[x, x] \neq 0$ for elements $x$ of odd degree (one can make the analogy more precise, but it is too much effort for a mere motivating example). Now look at the following graded Lie algebra: consider the graded set $X=\left\{x_{1}, x_{2}\right\}$, where the subscript represents the degree, and $L(X)$ the free graded rational Lie algebra generated by $X$. We quickly check that $L(X)$ admits the following basis in low dimensions:

(1) In word length 1: $x_{1}, x_{2}$;

(2) In length 2: $\left[x_{1}, x_{1}\right],\left[x_{1}, x_{2}\right]$;

(3) In length 3: [[ $\left.\left.x_{1}, x_{1}\right], x_{1}\right]$, [[ $\left.\left.x_{1}, x_{2}\right], x_{2}\right]$;

(4) In length 4: [[[ $\left.\left.\left.x_{1}, x_{1}\right], x_{2}\right], x_{2}\right],\left[\left[x_{1}, x_{2}\right],\left[x_{1}, x_{2}\right]\right]$.

The element which corresponds to item (4) in the twisted case was not detected by Barratt. So we may suspect it ought to be.

We already guess how to improve Barratt's intuition. To get a generating system, we have to consider the brackets $[\ldots[u, u] \ldots, u]$ not only for $u \in X$ but also for elements $u$ obtained from Lyndon words: $\left[\left[x_{1}, x_{2}\right],\left[x_{1}, x_{2}\right]\right]=2\left[x_{1}, x_{2}\right]^{2}$, where $\left[x_{1}, x_{2}\right]$ is obtained from the Lyndon word $x_{1} x_{2}$.

Our proof follows the classical one: the Lyndon words give an independent set, and the Klyachko idempotent proves that this set is generating. We study the Klyachko idempotent in the Hopf algebra environment: then the proofs work abstractly on morphisms and limit the complications involved by the action of the permutation group on words.

The paper is organized as follows.

We recall some definitions about twisted algebraic structures very briefly in Sect. 2. We also set up notation once for all.

Section 3 gives a short account on various notions of free twisted associative and Lie algebras. Some light is brought to associative and Lie polynomials in the twisted case.

In Sect. 4, we discuss Lyndon words and prove the minimality of our Hall basis.

Section 5 proves the properties of the Klyachko idempotent for Hopf algebras.

In Sect. 6, we prove that our basis is generating.

\section{Twisted algebras}

We briefly review some twisted algebraic structures we shall use in the following sections. A complete exposition was given by Stover [12]. We follow his presentation: it is very explicit on elements and so immediately manageable when we construct Hall basis.

First, we fix some notation for the permutation group.

\subsection{Permutation groups}

Let us denote by $\mathfrak{S}_{n}$ the group of all bijections of $n$ objects; in the following, it is understood (if the converse is not specified) that these objects are the set of integers $\{1, \ldots, n\}$; we also explicitly denote a permutation $\sigma$ by its image 
$(\sigma(1), \ldots \sigma(n))$. We compose permutations as usual for maps by acting on the left $\sigma \circ \tau(i)=\sigma(\tau(i))$.

Given a decomposition (all integers in the following are nonnegative) $\left(p_{1}, p_{2}\right)$ of $n=p_{1}+p_{2}$ (by abuse we shall say a decomposition $n=p_{1}+p_{2}$ ), we define the inclusion $\mathfrak{S}_{p_{1}} \times \mathfrak{S}_{p_{2}} \subset \mathfrak{S}_{n}$ as reflecting the inclusion given on objects by the map preserving order from left to right:

$$
\begin{aligned}
& \left\{1, \ldots, p_{1}\right\} \coprod\left\{1, \ldots, p_{2}\right\} \subset\{1, \ldots, n\}, \\
& i \mapsto i \text { on the first factor, } \\
& i \mapsto p_{1}+i \text { on the second factor. }
\end{aligned}
$$

(As above and by abuse, for $\bigsqcup$ order matters.) One immediately extends this to the case $n=p_{1}+\cdots+p_{k}, k \geq 2$, to define $\mathfrak{S}_{p_{1}} \times \cdots \times \mathfrak{S}_{p_{k}} \subset \mathfrak{S}_{n}$. If $\Phi_{i}$ are permutations in $\mathfrak{S}_{i}$, we denote then by $\left(\Phi_{1}, \ldots, \Phi_{k}\right)$ the image in $\mathfrak{S}_{n}$ of $\left(\Phi_{1} \times \cdots \times \Phi_{k}\right) \in \mathfrak{S}_{p_{1}} \times$ $\cdots \times \mathfrak{S}_{p_{k}}$.

We now define permutations acting on blocks. Let $n=p_{1}+\cdots+p_{k}$ be some decomposition and $\sigma \in \mathfrak{S}_{k}$. We define the permutation of $\mathfrak{S}_{n}$ acting on the $k$-blocks $p_{1}, \ldots, p_{k}$ by the following composition:

$$
\begin{gathered}
\mathcal{C}_{p_{\sigma^{-1}(1)}, \ldots, p_{\sigma^{-1}(k)}}(\sigma):\{1, \ldots, n\} \rightarrow\left\{1, \ldots, p_{1}\right\} \coprod \cdots \coprod\left\{1, \ldots, p_{k}\right\} \\
\rightarrow\left\{1, \ldots, p_{\sigma^{-1}(1)}\right\} \coprod \cdots \coprod\left\{1, \ldots, p_{\sigma^{-1}(k)}\right\} \rightarrow\{1, \ldots, n\}
\end{gathered}
$$

where the first and last arrows preserve the order from left to right, and the second one preserves the elements (i.e., if $\sigma(j)=i$, at the $l$ th spot of the $i$ th block of the image, you find the element that was at the $l$ th spot of the $j$ th block in the preimage).

We also recall the following:

\section{Proposition 2.1.1}

(1) For all $\sigma, \tau \in \mathfrak{S}_{k}$, we have

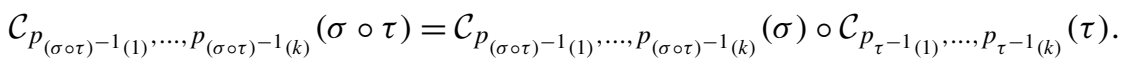

(2) For all $\sigma \in \mathfrak{S}_{k}, \Phi_{1} \in \mathfrak{S}_{p_{1}}, \ldots, \Phi_{k} \in \mathfrak{S}_{p_{k}}$, we have

$$
\mathcal{C}_{p_{\sigma^{-1}(1)}, \ldots, p_{\sigma^{-1}(k)}(\sigma) \circ\left(\Phi_{1} \times \cdots \times \Phi_{k}\right)=\left(\Phi_{\sigma^{-1}(1)} \times \cdots \times \Phi_{\sigma^{-1}(k)}\right)} \circ \mathcal{C}_{p_{\sigma^{-1}(1)}, \ldots, p_{\sigma^{-1}(k)}}(\sigma)
$$

2.2 Twisted modules and tensor products

Let $R$ be a ring. A graded $R$-module $X$ is a collection $\left(X_{n}\right)_{n \in \mathbb{N}}$ of $R$-modules $X_{n}$ indexed by the nonnegative integers. 
Twisted modules A twisted module $M$ is a graded module together with a right $\mathfrak{S}_{n}$-action (a right $R\left(\mathfrak{S}_{n}\right)$-module structure on $X_{n}$ for each $n$ ). Morphisms of graded $R$-modules and of twisted modules are defined as one can imagine; we shall only consider morphisms of degree 0 . A twisted module $M$ is connected if $M_{0}=0 . R$ is canonically given a structure of twisted module.

Twisted tensor product The twisted tensor product of $k$ twisted modules $M_{1}, \ldots, M_{k}$ is defined by its $n$th term

$$
\left(M_{1} \otimes \cdots \otimes M_{k}\right)_{n}=\sum_{\substack{p_{1}+\cdots+p_{k}=n \\ p_{i} \geq 0}}\left(\left(M_{1}\right)_{p_{1}} \otimes_{R} \cdots \otimes_{R}\left(M_{k}\right)_{p_{k}}\right) \otimes_{R\left(\mathfrak{S}_{p_{1}} \times \cdots \times \mathfrak{S}_{p_{k}}\right)} R\left(\mathfrak{S}_{n}\right) .
$$

2.3 Twisted algebras and coalgebras

With the definitions given above, we can formally define twisted algebras and twisted coalgebras by the same diagrams we do for the classical cases.

Like the classical case again we define the tensor twisted algebra $A \otimes B$ of two twisted algebras $A$ and $B$, the product of which is the composition

$$
A \otimes B \otimes A \otimes B \stackrel{A \otimes T \otimes B}{\longrightarrow} A \otimes A \otimes B \otimes B \stackrel{\mu_{A} \otimes \mu_{B}}{\longrightarrow} A \otimes B,
$$

which we can explicit on elements

$$
\left(\left(a_{1} \otimes b_{1}\right) \circ \sigma_{1}\right)\left(\left(a_{2} \otimes b_{2}\right) \circ \sigma_{2}\right)=\left(a_{1} a_{2}\right) \otimes\left(b_{1} b_{2}\right) \circ \mathcal{C}_{p_{1}, p_{2}, q_{1}, q_{2}}(T) \circ\left(\sigma_{1} \times \sigma_{2}\right) .
$$

We have denoted by $T$ the swap $A \otimes B \rightarrow B \otimes A$ and by $\sigma$ the permutation $(1,3,2,4)$.

\subsection{Twisted bialgebras, Hopf algebras}

We refer to [12] for the definitions of twisted algebras, coalgebras, and bialgebras. Formally they reproduce the definition diagrams of the classical case.

Definition 2.4.1 A twisted bialgebra is a twisted module $A$, which is both a twisted algebra with product $\mu: A \otimes A \rightarrow A$ and unit $\epsilon: R \rightarrow A$ and coalgebra with coproduct $\Delta: A \rightarrow A \otimes A$ and counit $\eta: A \rightarrow R$, such that both $\mu$ and $\eta$ are morphisms of twisted coalgebras or, equivalently, both $\Delta$ and $\epsilon$ are morphisms of twisted algebras; here $A \otimes A$ is given the structure of twisted coalgebra (resp. algebra) induced by $A$ and depicted in the preceding subsection.

Let us just emphasize the existence of the antipode in the axioms of Hopf algebras. 
Definition 2.4.2 A twisted Hopf algebra is a twisted bialgebra $A$ together with a morphism of twisted modules $S: A \rightarrow A$ such that the following diagram commutes:

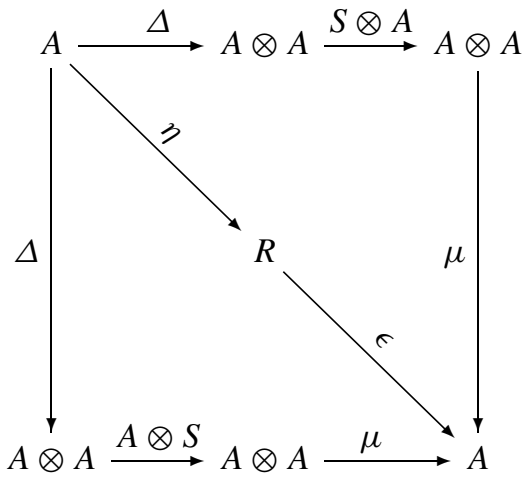

where $\eta: A \rightarrow R$ (resp. $\epsilon: R \rightarrow A$ ) is the counit (resp. unit) of the coalgebra (resp. algebra) $A$.

Convolution At this point, it seems judicious to introduce an operation we shall use very often in the next sections.

Proposition 2.4.3 Let $C$ be a twisted coalgebra, and A be a twisted algebra. The set of morphisms of twisted modules $\operatorname{Hom}_{R(\mathfrak{S})}(C, A)$ is an associative monoid with the following product, called the convolution and denoted by $\star$ :

$$
f \star g: C \stackrel{\Delta}{\longrightarrow} C \otimes C \stackrel{f \otimes g}{\longrightarrow} A \otimes A \stackrel{\mu}{\longrightarrow} A
$$

Now, by definition the antipode is the inverse of the identity under the convolution product; it is thus unique. Like in the classical case, there is a canonical way to define an antipode on a twisted connected bialgebra and thus to give it the structure of a twisted Hopf algebra.

Before continuing our description of twisted algebraic structures, let us recall the notion of pseudo-coproduct in cocommutative twisted bialgebras. We shall need it for the Klyachko idempotent (Sect. 5) and we already referred to it for the Dynkin idempotent in [1].

Let $A$ be a cocommutative bialgebra. We use the notation of Sect. 2 and denote by $\pi, \Delta, \eta$, and $\epsilon$ respectively its product, coproduct, unit, and counit. Let $\nu=\eta \circ \epsilon$. Formally, the same definition as in [9] works.

Definition 2.4.4 An endomorphism $f$ of $A$ (here and in the sequel, endomorphism means $\mathbb{F}(\mathfrak{S})$-module endomorphism, and we denote the corresponding set, $\mathbb{F}$-module, by $\operatorname{End}(A))$ admits $F \in \operatorname{End}(A \otimes A)$ as a pseudocoproduct if $F \circ \Delta=$ $\Delta \circ f$. If $f$ admits the pseudocoproduct $f \otimes v+v \otimes f$, we say that $f$ is pseudoprimitive. 


\subsection{Lie algebras}

Definition 2.5.1 A twisted Lie algebra $L$ is a twisted module together with a morphism of twisted modules $\beta: L \otimes L \rightarrow L$, called the bracket, which satisfies the traditional anticommutativity and Jacobi identities:

$$
\begin{aligned}
& \beta+\beta \circ T=0 \quad \text { in } \operatorname{Hom}_{R(\mathfrak{S})}(L \otimes L, L) \\
& \beta \circ(\beta \otimes L)+\beta \circ(\beta \otimes L) \circ(2,3,1)_{\#}+\beta \circ(\beta \otimes L) \circ(2,3,1)_{\#}^{2} \\
& \quad=0 \operatorname{Hom}_{R(\mathfrak{S})}(L \otimes L \otimes L, L)
\end{aligned}
$$

where $(2,3,1) \#$ acts on $L \otimes L \otimes L$ by $x \otimes y \otimes z \mapsto y \otimes z \otimes x$.

Let us be redundant and transcribe this definition on elements. As usual, we write the bracket $\beta=[$,$] , and the identities are written with explicit elements u_{i} \in L_{p_{i}}$ for $i=1,2,3$ :

$$
\begin{aligned}
& {\left[u_{1}, u_{2}\right]=\left[u_{2}, u_{1}\right] \circ \mathcal{C}_{p_{2}, p_{1}}((2,1))} \\
& {\left[\left[u_{1}, u_{2}\right], u_{3}\right]+\left[\left[u_{2}, u_{3}\right], u_{1}\right] \mathcal{C}_{p_{2}, p_{3}, p_{1}}((2,3,1))+\left[\left[u_{3}, u_{1}\right], u_{2}\right] \mathcal{C}_{p_{3}, p_{1}, p_{2}}((3,1,2))} \\
& \quad=0 .
\end{aligned}
$$

As in the classical case, we can define a Lie bracket on each twisted algebra $A$ by $\beta=\mu-\mu \circ T$ or on elements $[x, y]=x y-y x \mathcal{C}_{q, p}((2,1))$ for elements $x$ and $y$ of $A$ of respective degrees $p$ and $q$.

We conclude here the reminder on generalities about twisted algebraic structures. It gives a convenient framework to understand the notation of the coming sections. The paper of Stover [12] continues with enveloping algebras and the Milnor-Moore theorem.

Actually the theorem of Milnor-Moore also holds in the twisted context. Even if we shall need only part of the well-known results, let us recall some facts about primitive elements.

\subsection{Primitives in a twisted Hopf algebra}

If $A$ is a twisted bialgebra, an element $a \in A$ is primitive if $\Delta(a)=a \otimes 1+1 \otimes a$. The set of primitives of $A$ is a twisted submodule of $A$, denoted by $P A$.

Let us mention two results about $P A$ (cf. [12], Propositions 7.8 and 8.10).

Proposition 2.6.1 Consider $A$ as the twisted Lie algebra with bracket canonically induced by the (associative) product of A. Then PA is a twisted Lie subalgebra of $A$.

Proposition 2.6.2 If the Hopf algebra $A$ is cocommutative, then the inclusion PA $\subset$ $A$ induces an isomorphism of twisted Hopf algebras $U P A \cong A$. 
In the next section we wish to spread some light on various notions of free twisted objects. The most general one is defined by the usual process of adjunction (cf. [12]). Barratt [2], much more restrictive, defines an explicit basis in degree 1. Finally we shall extend the definition of [2] to generators of any degree: for that, we introduce the notion of twisted polynomials.

Let us now proceed and fix some ideas about free twisted objects.

\section{Free twisted objects and twisted Lie polynomials}

In this section we again follow Stover [12] and adapt the first chapter of Reutenauer's book [11] to the case of twisted structures.

First let us recall some basic definitions and properties of free monoids. Here no twisting occurs, and we shall be brief.

\subsection{Words and free monoids}

Let $X$ be a set, finite or infinite, and denote its elements by $x$ or $x_{i}$ for $i$ on some indexing set. A juxtaposition (or concatenation) of a finite number of ordered letters, e.g., $x_{1} x_{2} \ldots x_{n}$, is called a word. The collection of all words generated by $X$, denoted by $W(X)$, comes with an obvious embedding of sets $\imath: X \rightarrow W(X)$. Moreover $W(X)$ admits a product, called the concatenation product, defined as in the following example: $\left(x_{1} \ldots x_{n}\right)\left(x_{1}^{\prime} \ldots x_{n^{\prime}}^{\prime}\right)=x_{1} \ldots x_{n} x_{1}^{\prime} \ldots x_{n^{\prime}}^{\prime} . W(X)$ with this product is a free monoid. This definition is justified by the following:

Proposition 3.1.1 For any monoid $M$ and any map of sets $f: X \rightarrow M$, there is a unique map of monoids $\bar{f}: W(X) \rightarrow M$ such that the following diagram in the category of sets commutes:

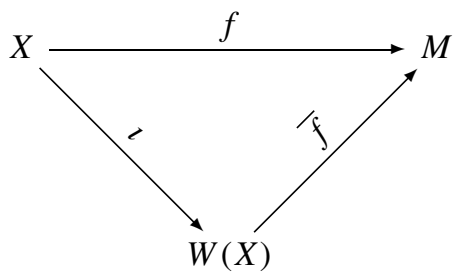

3.2 Definitions of free twisted objects and polynomials

Let $X$ be a graded set (each $x \in X$ is equipped with a positive integer $|x|$ called the degree), and $R$ be a ring. The twisted free module over $R$ generated by $X$ is any twisted module isomorphic to $\bigoplus_{x \in X} x R\left(\mathfrak{S}_{|x|}\right)$ and is denoted by $R(\mathfrak{S})(X)$. Again there is an obvious embedding of sets $\imath: X \rightarrow R(\mathfrak{S})(X)$.

Proposition 3.2.1 For any twisted module $M$ and any map of graded sets $f: X \rightarrow$ $M$, there is a unique map of twisted module $\bar{f}: R(\mathfrak{S})(X) \rightarrow M$ such that the follow- 
ing diagram in the category of graded sets commutes:

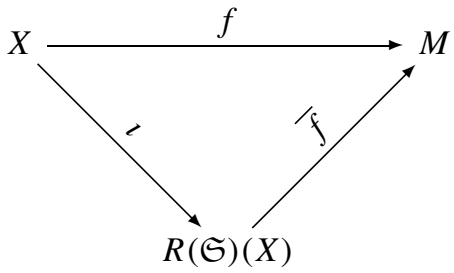

Proof Given any element $x_{1} r_{1}+\cdots+x_{n} r_{n}, x_{i} \in X, r_{i} \in R(\mathfrak{S})$, the commutation of the diagram implies that $\bar{f}\left(x_{i}\right)=f\left(x_{i}\right)$ and by linearity $\bar{f}\left(x_{1} r_{1}+\cdots+x_{n} r_{n}\right)=$ $f\left(x_{1}\right) r_{1}+\cdots+f\left(x_{n}\right) r_{n}$. Thus $\bar{f}$, if existing, is unique. Moreover the preceding formula is precisely a definition of $\bar{f}$ once $f$ is given.

Now let $M$ be a twisted module over $R$. Let us denote by $M^{\otimes n}$ the twisted module given by the tensor product of $n$ copies of $M$ and by $T(M)$ the direct sum $\bigoplus_{n>1} M^{\otimes n}$ (see Sect. 2.2 for the definition of the twisted tensor product). The associativity formula of Sect. 2.2 defines a (product) map $M^{\otimes n} \otimes M^{\otimes m} \rightarrow M^{\otimes(n+m)}$, which by linearity extends to $T(M)$ and endows it with a structure of an associative twisted algebra. This is called the free twisted (associative) algebra generated by the twisted module $M$. There is an obvious embedding of twisted modules $\imath: M \rightarrow T(M)$. This terminology is justified by the following:

Proposition 3.2.2 For any twisted (associative) algebra $A$ and any map of twisted modules $f: M \rightarrow A$, there is a unique map of twisted algebras $\bar{f}: T(M) \rightarrow$ A such that the following diagram in the category of twisted modules commutes:

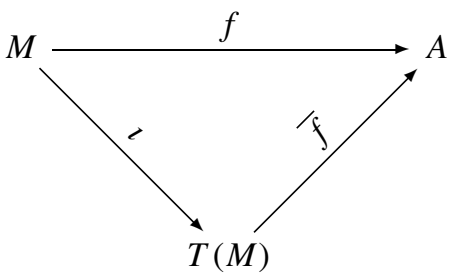

Proof Given an element $m_{1} \otimes \cdots \otimes m_{i} \otimes \sigma$, define $\bar{f}\left(m_{1} \otimes \cdots \otimes m_{i} \otimes \sigma\right)=$ $f\left(m_{1}\right) \otimes \cdots \otimes f\left(m_{i}\right) \otimes \sigma$. A straightforward inspection shows that $\bar{f}\left(m_{1} \sigma_{1} \otimes \cdots \otimes\right.$ $\left.m_{i} \sigma_{i} \otimes \sigma\right)=f\left(m_{1}\right) \sigma_{1} \otimes \cdots \otimes f\left(m_{i}\right) \sigma_{i} \otimes \sigma=f\left(m_{1}\right) \otimes \cdots \otimes f\left(m_{i}\right)\left(\sigma_{i} \times \sigma_{1} \times\right.$ $\left.\cdots \times \sigma_{i}\right) \sigma$. This proves, first, that $\bar{f}$ is well defined on $T(M)$ as a twisted module map and, secondly, that $\bar{f}$ is multiplicative. Moreover, by definition, $\bar{f}=f$ on the twisted module $M$. This completes the proof.

We briefly pause here to emphasize an important point we shall only use in the next subsection. Consider the map of twisted modules $\Delta: M \rightarrow T(M) \otimes T(M)$ given by $\Delta(m)=m \otimes 1+1 \otimes m$ and extend it to obtain a map of twisted algebras $\Delta$ : $T(M) \rightarrow T(M) \otimes T(M)$. This process endows $T(M)$ with a structure of twisted bialgebra. Actually this bialgebra is connected; indeed it is easy to check that the antiautomorphism $S: T(M) \rightarrow T(M)$ defined by $S(m)=-m$ (just apply the universal 
property of Proposition 3.2.2 to the algebra opposite to $T(M)$ ) satisfies the axioms of an antipode for $T(M)$. In other words we just defined the structure of twisted Hopf algebra for $T(M)$.

Now let us specialize to the free twisted module generated by a graded set $X$. Let us denote by $\mathcal{F}(X)$ the free twisted associative algebra $T(R(\mathfrak{S})(X))$. Combining Propositions 3.2.1 and 3.2.2, we readily obtain the following:

Proposition 3.2.3 For any twisted associative algebra $A$ and any map of graded sets $f: X \rightarrow A$, there is a unique map of twisted algebras $\bar{f}: \mathcal{F}(X) \rightarrow A$ such that the following diagram in the category of graded sets commutes:

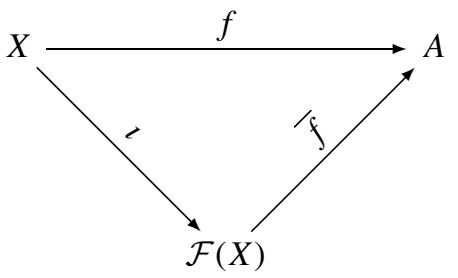

We end this subsection by introducing polynomials in the twisted case. A typical element of $R(\mathfrak{S})(X)$ ) may be written as $\sum_{i \in I} x_{i} \circ \sigma_{i}$, for a finite indexing set $I$. Thus $\mathcal{F}(X)$ is linearly generated (i.e., as an $R(\mathfrak{S})$-module) by elements of the type $\bigotimes_{j \in J} x_{j}$, where $J$ browses all finite tuples of elements of $X$. Such an element is also written $x_{1} \ldots x_{j}$ for a $j$-uple $\left(x_{1}, \ldots, x_{j}\right)$ and is called a monomial of $\mathcal{F}(X)$. The collection of monomials is a linear basis for $\mathcal{F}(X)$. In the algebra $\mathcal{F}(X)$, the product of polynomials follows the rules of the product in a free twisted algebra edicted in Sect. 2.2:

$$
\begin{aligned}
&\left(\left(x_{1,1} \sigma_{1,1} \otimes \cdots \otimes x_{1, k} \sigma_{1, k}\right) \tau_{1} \times\left(x_{2,1} \sigma_{2,1} \otimes \cdots \otimes x_{2, l}\right) \sigma_{2, l}\right) \tau_{2} \\
&=\left(\left(x_{1,1} \sigma_{1,1} \otimes \cdots \otimes x_{1, k} \sigma_{1, k}\right) \otimes\left(x_{2,1} \sigma_{2,1} \otimes \cdots \otimes x_{2, l} \sigma_{2, l}\right)\right) \tau_{1} \times \tau_{2} \\
&=\left(x_{1,1} \otimes \cdots \otimes x_{1, k} \otimes x_{2,1} \otimes \cdots \otimes x_{2, l}\right)\left(\sigma_{1,1} \times \cdots \times \sigma_{1, k} \times \sigma_{2,1} \times \cdots \times \sigma_{2, l}\right) \\
& \quad \times\left(\tau_{1} \times \tau_{2}\right) .
\end{aligned}
$$

\subsection{Free twisted Lie algebras and Lie polynomials}

We refer here to Stover [12], especially for the proofs.

Consider a nonassociative abstract operation on symbols and write it as a bracketing. Starting with a unique symbol, say $x$, the bracketing operation gives rise to an infinite set $\mathcal{N}(x)$, the free nonassociative monoid generated by $x$. Given a twisted module $M$ and an element $b$ of $\mathcal{N}(x)$, we define $M^{\otimes b}$ as the twisted module $M^{\otimes \# b}$, where $\# b$ denotes the number of occurrences of $x$ in $b$, and the twisted structure is similar to the twisted structure of the ordinary tensor product. The bracketing operation in the monoid $\mathcal{N}(x)$ induces an obvious bracketing operation $M^{\otimes b} \otimes M^{\otimes c} \rightarrow M^{\otimes(b c)}$. Let us define the twisted module $\mathcal{T}(M)=\bigoplus_{b \in \mathcal{N}(x)} M^{\otimes b}$. The bracketing operation just defined extends to $\mathcal{T}(M)$ by linearity. Call it $\beta$. 
Define $\mathcal{I}(M)$ as the two-sided twisted ideal of $\mathcal{T}(M)$ generated by the images of

$$
\begin{aligned}
& \beta+\beta \circ(2,1): \mathcal{T}(M) \times \mathcal{T}(M) \rightarrow \mathcal{T}(M) \\
& \beta \circ(\beta \times \mathcal{I}(M))+\beta \circ(\beta \times \mathcal{I}(M))(2,3,1)+\beta \circ(\beta \times \mathcal{I}(M))(2,3,1)^{2}: \\
& \quad \mathcal{T}(M) \times \mathcal{T}(M) \times \mathcal{T}(M) \rightarrow \mathcal{T}(M)
\end{aligned}
$$

The quotient $\mathcal{L}(M)=\mathcal{T}(M) / \mathcal{I}(M)$ is equipped with the map induced by $\beta$ (denoted as usual by [,]) and is called the free twisted Lie algebra generated by $M$, denomination justified by the following proposition proved by Stover [12]. There is an obvious embedding of twisted modules $\imath: M \rightarrow \mathcal{L}(M)$.

Proposition 3.3.1 For any twisted Lie algebra $L$ and any map of twisted modules $f: M \rightarrow L$, there is a unique map of twisted Lie algebras $\bar{f}: \mathcal{L}(M) \rightarrow L$ such that the following diagram in the category of twisted modules commutes:

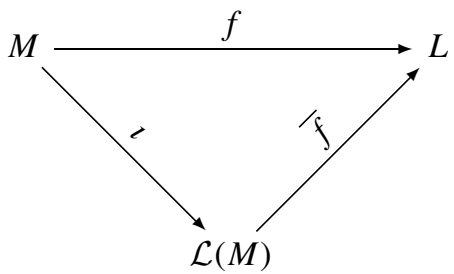

Let us end this subsection with some lines about twisted universal enveloping algebras.

If $L$ is a twisted Lie algebra, consider the free (associative) twisted algebra $T(L)$ generated by the twisted module $L$ with the linear embedding $l: L \rightarrow T(L)$. Now let $I L$ be the two-sided twisted Lie ideal generated in $T(L)$ by the elements of the form $[\imath(x), \imath(y)]-\imath[x, y]$.

The enveloping algebra of $L$ is the quotient (associative) algebra $T(L) / I(L)$ and is denoted by $U L$. It satisfies the following:

Proposition 3.3.2 For any Lie algebra $L$ and any map of Lie algebras $f: L \rightarrow A$, there is a unique map of algebras $\bar{f}: U L \rightarrow A$ such that the following diagram in the category of sets commutes:

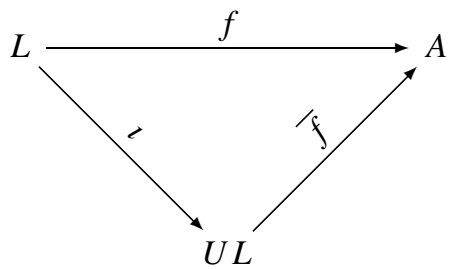

Now we can phrase the twisted version of the Milnor-Moore theorem for free algebras given in [12], Proposition 7.4. 
Theorem 3.3.3 Let $M$ be a twisted module. The twisted algebra map

$$
T(M) \rightarrow U \mathcal{L}(M)
$$

induced by the composition of maps of twisted modules

$$
M \rightarrow \mathcal{L}(M) \rightarrow U \mathcal{L}(M)
$$

is an isomorphism.

As in the preceding subsection, we can introduce Lie polynomials. Recall that a typical element of $R(\mathfrak{S})(X)$ may be written as $\sum_{i \in I} x_{i} \circ \sigma_{i}$ for an indexing set $I$ and that a (noncommutative) polynomial in $\mathcal{F}(X)$ is a linear combination of elements such as $x_{1} \sigma_{1} \otimes \cdots \otimes x_{j} \sigma_{j}$. By Sect. 2.4 and Proposition 3.3.1 we define an embedding of twisted Lie algebras, $\mathcal{L}(X) \subset \mathcal{F}(X)$. A polynomial in $\mathcal{F}(X)$ is called a Lie polynomial if it is in the image of $\mathcal{L}(X)$ by this embedding.

Remark If we suppose that all elements of $X$ are of degree 1, we recover Barratt's definition of a free twisted (associative) algebra and Lie algebra.

\section{Lyndon words}

Preliminary remark One can ask-and we are grateful to the referee for his question-why we are limited to Lie algebras over free twisted modules. The reason rests on the next two sections. As the classical one (see [4]), our proof uses Lyndon words. Let us have an idea of the problem. Suppose that $M$ is defined on the rationals by two generators $x$ and $y$ of degree 2 and a relation $x(1+\epsilon)=y(1-\epsilon)$, where $\epsilon=(2,1) \in \mathbb{Q}\left(\mathfrak{S}_{2}\right)$ (notice that $1-\epsilon$ and $1+\epsilon$ are zero divisors in $\mathbb{Q}\left(\mathfrak{S}_{2}\right)$ and the set of generators $\{x, y\}$ is minimal). Of course this relation induces further relations between all monomials in $x$ and $y$, and the fundamental Theorem 4.2.2 is no more valid.

This section (and Sect. 5) follows the presentation of [4] in outline. We have now to handle carefully the twisted structures. We have precisely in mind that for an element $u$ in a twisted Lie algebra, the bracket $[\ldots[u, u], \ldots, u]]$ is not necessarily 0 . So, when building a basis, we have to modify the classical definitions and to check properties again. Let us proceed for Lyndon words.

\subsection{Free twisted associative algebra context}

First we fix a basis field $\mathbb{F}$ of characteristic 0 . Let also $X$ be a graded set; we denote its elements by $x_{i}$. Let us denote by $W(X)$ the set of words in $X$; the length of a word is the number of elements of $X$ necessary to write it down by concatenation; conventionally 1 is the word of length 0 . Write $\mathcal{F}(X)$ for the free associative twisted algebra generated by $X$ (cf. Sect. 3); as an $\mathbb{F}(\mathfrak{S})$-module, $\mathcal{F}(X)$ admits $W(X)$ for basis. As 
usual, the product in $\mathcal{F}(X)$ is denoted by the simple juxtaposition $f g$, and so the canonical Lie algebra structure on $\mathcal{F}(X)$ is given by $[f, g]=f g-g f \mathcal{C}_{|g|,|f|}((2,1))$.

Finally let us write $\mathcal{L}(X)$ for the free twisted Lie algebra generated by $X$, which we consider as a Lie twisted subalgebra of $\mathcal{F}(X)$ with its canonical Lie algebra structure.

We now define Lyndon words of $\mathcal{F}(X)$ in various equivalent ways and examine how using them to obtain generators of $\mathcal{L}(X)$.

Let $u, v, w$ be words (elements of $W(X))$ of strictly positive length such that $w=$ $u v$. We say that $u$ is a head of $w$ and that $v$ is a tail of $w$. We order words of $W(X)$ by lexicographic order and denote the order relation by $\geq$. In particular, if $w \geq u v$, either the order is decided in $u$ and $w \geq u$, or only in $v$, and then $u$ is a head of $w$.

Definition 4.1.1 A Lyndon word is a word that is strictly smaller than all its cyclic rearrangements or a power not less than 2 of a Lyndon word.

Remark The preceding definition makes sense because it is recursive. Clearly all elements of $X$ are Lyndon words (these are all Lyndon words of length 1 -like in the nontwisted case), and if $w=u^{p}, p \geq 2$, the length of $u$ is strictly smaller that the length of $w$.

We fix now some notation. Let $L$ (resp. $L_{n}$ ) denote all Lyndon words of $\mathcal{F}(X)$ (resp. of length $n$ ).

We say that a word $w=x_{1} x_{2} \ldots x_{k}$ is not prime if there is some nontrivial circular permutation $\sigma \in \mathfrak{S}_{k}$ such that $w=x_{\sigma(1)} x_{\sigma(2)} \ldots x_{\sigma(k)}$. In the opposite case we say that $w$ is prime.

Proposition 4.1.2 $w$ is a Lyndon word if and only if

(1) If $w$ is prime, then $w$ is strictly smaller than all its tails, or

(2) If $w$ is not prime, then there exists a Lyndon word $u$ such that $w=u^{p}, p>1$.

Proof $(\Leftarrow)$ Suppose that $w$ is smaller than all its tails. Write $w=u v$ with $u$ and $v$ of strictly positive length. Then $w<v$, which implies $w<v u$. As $v$ is a tail of $w$, this means that $w$ is strictly smaller than all its cyclic rearrangements.

$(\Rightarrow)$ Let $w=\alpha v$, with $\alpha$ and $v$ of strictly positive length. Then $w<v \alpha$.

(a) Either this inequality is decided in $v$, and we are done,

(b) or $v$ is a head of $w$, and $w=v \beta$; then by hypothesis

(1) $w=\alpha v<\beta v$, and thus $\alpha<\beta$,

(2) and vice-versa: $w=v \beta<v \alpha$, and thus $\beta<\alpha$,

which shows that (b) cannot happen.

Proposition 4.1.3 $w$ is a Lyndon word if and only if it has a factorization:

$$
\text { If } w \text { is prime, } w=w_{1} w_{2} \text { with } w_{1}, w_{2} \in L \text { and } w_{1}<w_{2} \text {, }
$$

or

$$
\text { If } w \text { is not prime, } w=u^{p}, p>1, u \text { a prime Lyndon word. }
$$


Moreover if $w$ is prime and $w_{2}$ is the longest possible Lyndon word, $w_{1}$ and $w_{2}$ are prime.

Proof $\Rightarrow$

Let $w=w_{1} w_{2}$ with $w_{2}$ the longest Lyndon tail of $w$. We shall first show that $w_{1}$ is strictly smaller than $w_{2}$ and secondly that $w_{1} \in L$, which means that the decomposition matches the request.

By Proposition 4.1.2, $w=w_{1} w_{2}<w_{2}$. Suppose $w_{1} \geq w_{2}$; then $w_{1} u \geq w_{2}$ for every word $u$, in particular $u=w_{2}$, which contradicts our hypothesis; so $w_{1}<w_{2}$. Our first assertion holds.

Let us prove now that $w_{1}$ is a Lyndon word.

First examine the two possible cases.

(A) $w_{1}$ is a prime word.

We use Proposition 4.1.2 again and show that $w_{1}$ is strictly smaller than all its tails. Decompose $w_{1}=u v$ with $u$ and $v$ of strictly positive length.

$w=u v w_{2}$, and our choice of $w_{2}$ implies that $v w_{2}$ cannot be a Lyndon word. So, by Proposition 4.1.2, there exists a decomposition $v w_{2}=s t$ with $t<v w_{2}$.

(a) Either this inequality is decided in $v: v>t$. Going back to $w$, we get $w=u s t$ and $t>w=w_{1} w_{2}>w_{1}$, and we deduce that $v>t>w_{1}$, as desired.

(b) Or $v$ is a head of $t$, and we can write $t=v s^{\prime}$; then $v w_{2}=s t=s v s^{\prime}$. In other words, $s^{\prime}$ is a tail of $w_{2}$. Since $w_{2}$ is a Lyndon word:

(i) Either $w_{2}<s^{\prime}$, and we derive

$$
v w_{2}>t=v s^{\prime}>v w_{2}
$$

which is a contradiction.

(ii) Or $w_{2}=u^{\prime p}, p>1$, with $u^{\prime}$ a prime Lyndon word (equivalently, $p$ maximal). If $s^{\prime}=s^{\prime \prime} u^{\prime k}$ with $s^{\prime \prime}$ of length strictly positive but smaller than the length of $u^{\prime}$. Then $s^{\prime \prime}$ is a tail of $u^{\prime}$. As $u^{\prime}$ is a prime Lyndon word, $u^{\prime}<s^{\prime \prime}$.

Besides,

$$
v u^{\prime p}=v w_{2}>t=v s^{\prime \prime} u^{\prime k} .
$$

Since the length of $s^{\prime \prime}$ is strictly smaller than the length of $u^{\prime}$, this forces

$$
u^{\prime}>s^{\prime \prime}
$$

in contradiction with the above assertion.

So the case $w_{2}=u^{\prime p}$ cannot occur.

(B) $w_{1}$ is not prime, say $w_{1}=(u v)^{p}, p>1$.

Then $w=(u v)^{p} w_{2}$. As $w$ is a Lyndon word, our choice for $w_{2}$ implies that $v w_{2}$ is not a Lyndon word. Thus there exists a decomposition $v w_{2}=s t$, with $t<v w_{2}$.

(a) Either this inequality is decided in $v$ and $v>t$. Then

$$
w=(u v)^{p-1} u v w_{2}=(u v)^{p-1} u s t
$$

and, as $w$ is a Lyndon word with tail $t$,

$$
t>(u v)^{p-1} u s t>u v .
$$


Thus,

$$
v>t>u v
$$

which proves that $u v$ is a Lyndon word.

Now

$$
u v<(u v)^{p}<w_{2}
$$

by the first part of the proof. Thus $u v w_{2}$ is a product of two Lyndon words $u v$ and $w_{2}$ with $u v<w_{2}$. If we suppose recursively that the theorem holds in length smaller than the length of $|w|$, we conclude that $u v w_{2}$ is a Lyndon word, a contradiction with our hypothesis on $w_{2}$.

(b) Or the inequality is not decided in $v$, and $v$ is a head of $t$. Then we can reproduce the argument in (A), case (b), because in this part of the proof we do not use that $w$ is a Lyndon word, only the fact that $w_{2}$ is one.

So the study of case (B) proves that this case is, in fact, impossible: $w_{1}$ is necessarily a prime word.

$\Leftarrow$

We use Proposition 4.1.2 once more and give ourselves a decomposition $w=u v$. We begin with the case where $v=w_{2}$ (and so $u=w_{1}$ ). By hypothesis, $w_{1}<w_{2}$. If this inequality is decided before the end of $w_{1}$, then $w_{1} \beta<w_{2}$ for every word $\beta$. If not, this means that $w_{1}$ is a head of $w_{2}$, and then we can write $w_{2}=w_{1} \alpha$. Now $w_{2}$ is a Lyndon word and $w_{2}<\alpha$, which implies $w=w_{1} w_{2}<w_{1} \alpha=w_{2}$.

If $v$ is shorter than $w_{2}$, then $v$ is a tail $w_{2}$, and (always Proposition 4.1.2) $v>$ $w_{2}>w$.

If $v$ is longer than $w_{2}, w_{2}$ is a tail $v: v=s w_{2}$ for some word $s$. Then $s$ is a tail of $w_{1}$. Now $w_{1}$ is also a Lyndon word; thus $w_{1}<s$, which implies $w=w_{1} w_{2}<$ $s w_{2}=v$. This was to be proved.

Definition 4.1.4 A standard factorization of a Lyndon word $w$ is a factorization of one of the two types:

(i) $w=w_{1} w_{2}$, where $w_{1}$ and $w_{2}$ are Lyndon words, $w_{1}<w_{2}$ with $w_{2}$ the longest word possible, or

(ii) $w=u^{p}, u$ a Lyndon word, and $p>1$ with the greatest $p$ possible.

\subsection{Free twisted Lie algebra context}

In this section we explain how to use the Lyndon words to construct a basis of the free twisted Lie algebra.

Let us define a map $b: L \rightarrow \mathcal{L}(X)$. We start with $x \in X: b(x)=x \in \mathcal{L}(X)$. Then the definition is recursive: if $w=w_{1} w_{2}$ (factorization (i) of Definition 4.1.4) we set $b(w)=\left[b\left(w_{1}\right), b\left(w_{2}\right)\right]$; if $w=u^{p}$ (factorization (ii) of Definition 4.1.4), we set $b(w)=[[b(u), b(u)], \ldots, b(u)]$.

We are now ready to show how Lyndon words generate an independent set in $\mathcal{L}(X)$. 
Proposition 4.2.1 If $w$ is a prime Lyndon word, then:

$$
b(w)=w+\sum_{v>w} v a_{v} \quad \text { with } a_{v} \in R(\mathfrak{S}) .
$$

If $w=u^{p}$, then:

$$
b(w)=(b(u))^{p}\left(1-\gamma_{2}\right) \ldots\left(1-\gamma_{p}\right),
$$

where $\gamma_{i}$ is the permutation of $\mathcal{C}_{(i-1)|u|,|u|}((2,1))$ of $\mathfrak{S}_{i|u|}$.

Proof We begin with the case $w=u^{p}$. By recursion it is enough to prove that $b(w)=$ $b\left(u^{p-1}\right) b(u)\left(1-\gamma_{p}\right)$. Now $b\left(u^{p}\right)=\left[b\left(u^{p-1}\right), b(u)\right]$ by the definition of $b$; then apply the definition of the twisted Lie bracket in $\mathcal{F}(X)$.

Let us now examine the prime case. Again we proceed recursively. So we have the standard factorization $w=w_{1} w_{2}$, and by the hypothesis of recursion we can write when $w_{1}$ and $w_{2}$ are prime:

$$
b(w)=w_{1} w_{2}-w_{2} w_{1} \mathcal{C}_{\left|w_{1}\right|,\left|w_{2}\right|}((2,1))+\sum\left(v_{1} v_{2}-v_{2} v_{1} \mathcal{C}_{\left|v_{1}\right|,\left|v_{2}\right|}((2,1))\right),
$$

where the sum is over all pairs $\left(v_{1}, v_{2}\right)$ where $v_{i}$ appears in the decomposition of $w_{i}$ excepting the pair $\left(w_{1}, w_{2}\right)$. So $v_{1} v_{2}>w_{1} w_{2}$ because either $v_{1}>w_{1}$ or if $v_{1}=w_{1}$, then $v_{2}>w_{2}$. Similarly, $v_{2} v_{1}>w_{2} w_{1}$, and $w_{2} w_{1}>w_{1} w_{2}$ because $w_{1} w_{2}=w$ is a Lyndon word.

If $w_{1}=u^{p}$ or $w_{2}=v^{q}$, then the same argument holds.

The last discussion leads immediately to the fundamental result:

Theorem 4.2.2 The polynomials $\{b(w)\}_{w \in L}$ are independent in $\mathcal{F}(X)$.

We want now to prove that these polynomials are generating. As in the classical case, the major tool is the Klyachko idempotent.

\section{The Klyachko idempotent}

We follow here the presentation of [9] and work in a bialgebra. We shall specialize to the Lie algebra in the next section.

Let $A$ be a twisted bialgebra, and $\mathbb{F}$ be a field of characteristic 0 , which contains a primitive $n$th root of the unity $\omega_{n}$ for any $n \geq 1$. Let $p_{n}: A \rightarrow A_{n} \hookrightarrow A$ be the projection of $A$ onto its component of degree $n$ (viewed as a morphism of $\operatorname{End}_{\mathbb{F}(\mathfrak{S})}(A)$ ) and define $p_{C}=p_{i_{1}} \star \cdots \star p_{i_{l}}$, where $C$ denotes the $l$-uple of strictly positive integers $\left(i_{1}, \ldots, i_{l}\right)$; we shall also say that $C$ is a composition of $\left(i_{1}+\cdots+i_{l}\right)$. By definition $C$ is finer than $C^{\prime}$, and we write $C^{\prime} \leq C$ if $C^{\prime}$ is obtained from $C$ by substituting to a subset of consecutive entries of $C$ (say $i_{k}, i_{k+1}, \ldots, i_{k+l}, 1 \leq k \leq k+l \leq j$ ) their sum $\left(i_{k}+\cdots+i_{k+l}\right)$; notice that this substitution does not change the total sum of all entries of $C$, which we call the weight; see below. 
By inclusion-exclusion we define the elements $r_{C}$ of $\operatorname{End}_{\mathbb{F}(\mathfrak{S})}(A)$ by the formula

$$
p_{C}=\sum_{C^{\prime} \leq C} r_{C^{\prime}}
$$

More precisely, let $l(C)$ be the length - the number of entries-of $C$. Then (by Moebius inversion)

$$
r_{C}=\sum_{C^{\prime} \leq C}(-1)^{l\left(C^{\prime}\right)-l(C)} p_{C^{\prime}}
$$

For any $l$-uple $C=\left(i_{1}, \ldots, i_{j}\right)$, define its weight by $|C|=i_{1}+\cdots+i_{l}$ and its major index by $\operatorname{maj}(C)=(l-1) i_{1}+(l-2) i_{2}+\cdots+i_{l-1}$. With this notation let us define:

Definition 5.0.3 The Klyachko idempotent-this denomination will be justified below-of order $n$ is the morphism $\kappa_{n} \in \operatorname{End}_{\mathbb{F}(\mathfrak{S})}(A)$ given by the formula

$$
\kappa_{n}=\frac{1}{n} \sum_{|C|=n} \omega_{n}^{\operatorname{maj}(C)} r_{C} .
$$

Theorem 5.0.4 If $A$ is a cocommutative, connected bialgebra, then $\kappa_{n}$ maps $A$ into the primitives of the bialgebra $A$.

Proof We reproduce the proof of [9] and use the shortcut presented in [5]. A priori we have to pay attention to the action of $\mathfrak{S}$, in particular when dealing with tensor products.

Actually the general presentations by morphisms [9] veils the effective action of S: the abstract formulas for structure maps of the twisted bialgebra $A$ do not involve permutations explicitly; they only appear when we want to make them explicit on elements of $A$.

We define $\operatorname{Endgr}(A)=\bigoplus_{n>0} \operatorname{End}_{\mathbb{F}\left(\mathfrak{S}_{n}\right)}\left(A_{n}\right)$. Let $q$ be a variable; then $\operatorname{Endgr}(A)[[q]]$ makes sense. As the morphisms of $\operatorname{End}_{\mathbb{F}(\mathfrak{S})}(A)$ are of degree 0 , there is a bijection between $\operatorname{End}(A)$ and $\operatorname{Endgr}(A)$ compatible with the action of $\mathbb{F}\left(\mathfrak{S}_{n}\right)$; so we can transfer the convolution product to $\operatorname{Endgr}(A)$. Define $P(q)=\sum_{n \geq 0} p_{n} q^{n} \in$ $\operatorname{Endgr}(A)$. The infinite product

$$
\kappa(q)=\cdots \star P\left(q^{n}\right) \star \cdots \star P(q) \star P(1)
$$

is well defined in $\operatorname{Endgr}(A)[[q]]$, because $A$ is connected.

Observe that each element of $\operatorname{Endgr}(A)[[q]]$ has a unique expression as a sum $\sum_{n} f_{n}$, with $f_{n} \in \operatorname{End}\left(A_{n}[[q]]\right)$. Like in [9] (after [5]), these elements can be easily deduced from the formula

$$
\kappa(q)=\sum_{n \geq 0} \frac{K_{n}(q)}{(q)_{n}}
$$

with $(q)_{n}=(1-q) \ldots\left(1-q^{n}\right)$ and $K_{n}(q)=\sum_{|C|=n} q^{\operatorname{maj}(C)} r_{C}$. Notice that in the above formula $n$ actually is the degree involved in $\operatorname{Endgr}(A)$. 
We extend the definition of pseudo-coproduct recalled in Sect. 2 and say that $f \in$ $\operatorname{Endgr}(A)[[q]]$ admits the pseudo-coproduct $F \in \operatorname{Endgr}(A) \otimes \operatorname{Endgr}(A)[[q]]$ if $F$ 。 $\delta=\delta \circ f$, where $\delta$ extends naturally to $A[[q]]$. Moreover, there is a natural bijection (compatible with the action of the $\mathfrak{S}_{n}$ ) between Endgr $(A)[[q]]$ and $\mathfrak{S}$-morphisms $A \rightarrow A[[q]]$ (similarly between $\operatorname{Endgr}(A) \otimes_{\mathbb{F}} \operatorname{Endgr}(A)[[q]]$ and morphisms $A \otimes_{\mathbb{F}}$ $A \rightarrow A \otimes_{\mathbb{F}} A[[q]]$. We systematically identify $\operatorname{Endgr}(A) \otimes_{\mathbb{F}[[q]]} \operatorname{Endgr}(A)[[q]]$ with $\left(\operatorname{Endgr}(A) \otimes_{\mathbb{F}} \operatorname{Endgr}(A)\right)[[q]]$. Granting this, for $f, g \in \operatorname{Endgr}(A)[[q]]$, we consider $f \otimes g$ as an element of $\left(\operatorname{Endgr}(A) \otimes_{\mathbb{F}} \operatorname{Endgr}(A)\right)[[q]]$. With all these conventions, Theorem 5.0.8 of [1] applies, since we assumed $A$ to be cocommutative.

We check that $\sum_{i+j=n} p_{i} \otimes p_{j} \circ \delta=\delta \circ p_{n}$, i.e., $\sum_{i+j=n} p_{i} \otimes p_{j}$ is a pseudocoproduct for $p_{n}$ (this is general; if $f=\sum f_{n}, f \otimes f$ is a pseudo-coproduct for $f$ if and only if $\sum_{i+j=n} f_{i} \otimes f_{j}$ is a pseudo-coproduct for $f_{n}$ ). This is equivalent to say that $P(q) \otimes P(q)$ is a pseudo-coproduct for $P(q)$ (the same is true for $P\left(q^{n}\right)$ for any $n>0)$. Then, applying Theorem 5.0.8 of [1], we deduce that $\kappa(q) \otimes \kappa(q)$ is a pseudocoproduct for $\kappa(q)$. By the above result, this means that $\sum_{i+j=n} \frac{K_{i}(q)}{(q)_{i}} \otimes \frac{K_{j}(q)}{(q)_{j}}$ is a pseudo-coproduct for $\frac{K_{n}(q)}{(q)_{n}}$, or equivalently $\sum_{i+j=n} \frac{(q)_{n}}{(q)_{i}(q)_{j}} K_{i}(q) \otimes K_{j}(q)$ is a pseudo-coproduct for $K_{n}(q)$. The polynomials $\frac{(q)_{n}}{(q)_{i}(q)_{j}}$ vanish for $q=\omega_{n}$, except the cases where $i=0$ or $j=0$ (in both cases they are equal to 1 ). This means that $K_{n}\left(\omega_{n}\right)=n \kappa_{n}$ is pseudo-primitive and proves the theorem.

Corollary 5.0.5 If $A$ is a cocommutative, connected bialgebra, then $\kappa_{n}$ is an idempotent.

Proof For sake of completeness, we reproduce the proof of [4]. Here there are no changes introduced by the action of $\mathfrak{S}$.

First notice that the coproduct $\delta$ of the bialgebra $A$ preserves the degree; therefore $p_{C}$ is 0 on all elements of $A$ of degree not equal to the length of $C$ (just consider the coassociativity of the coproduct, which implies the associativity of the convolution). So, by the previous theorem and since $A-$ a cocommutative and connected bialgebra-is generated by its primitive elements (Proposition 2.6.2), it is enough to prove that $\kappa_{n}(a)=a$ for any primitive element $a \in A$; by the preceding remark there is no restriction to suppose that $|a|=n$. As $a$ is primitive, $p_{i} \star p_{j}(a)=0$, and $p_{C}(a) \neq 0$ only if $C$ is of length 1 . Equation (1) implies that $r_{C}(a)=(-1)^{l(C)-1} a$ for each $C$ of weight $n$. Thus,

$$
n \kappa_{n}(a)=\sum_{|C|=n} \omega_{n}^{\operatorname{maj}(C)}(-1)^{l(C)-1} a .
$$

Now there is classical bijection between compositions of $n$ and subsets of $\{1, \ldots$, $n-1\}$, sending $S=\left(i_{1}, \ldots, i_{l}\right)$ onto $S=\left\{i_{1}, i_{1}+i_{2}, \ldots, i_{1}+\cdots+i_{l-1}\right\}$. The cardinality of $S$ is $l(C)-1$, and we define $\operatorname{maj}(S)=\sum_{i \in S} i=\operatorname{maj}(C)$. With this notation,

$$
\begin{aligned}
n \kappa_{n}(a) & =\sum_{S \subset\{1, \ldots, n-1\}} \omega_{n}^{\operatorname{maj}(S)}(-1)^{\operatorname{card}(S)} a \\
& =\sum_{1 \leq i_{1}<\cdots<i_{r} \leq n-1} \omega_{n}^{i_{1}+\cdots+i_{r}}(-1)^{r} a
\end{aligned}
$$




$$
\begin{aligned}
& =\prod_{1 \leq i \leq n-1\}}\left(1-\omega_{n}^{i}\right) a \\
& =(1+1+\cdots+1) a=n a
\end{aligned}
$$

since $\omega_{n}$ is a primitive $n$-root of the unity.

\section{The Lyndon-Hall basis}

This section tells how to use the Klyachko idempotent for proving that the Lyndon words are generating.

We proved in Corollary 5.0.5 that $\kappa_{n}$ is the identity on primitives of the bialgebra $\mathcal{F}(X)$. Moreover, $\kappa_{n}$ maps $\mathcal{F}(X)$ into its primitive elements, and thus, by the Theorem 3.3.3 of Milnor-Moore, we conclude that $\kappa_{n}(\mathcal{F}(X))=\mathcal{L}(X)$.

In Theorem 4.2.2 we proved that the set $L$ of Lyndon words determines a minimal set of independent elements in $\mathcal{L}(X)$. In this section we want to prove that this set is generating (actually this is directly related to the fact that, by definition, $L$ forms a set of representatives of all circular rearrangements classes of words of $W(X))$. Let us see that.

To this purpose, we shall use the Klyachko idempotent $\kappa_{n}$. The following theorem tells us that $\kappa_{n}$ does not discriminate between all circular rearrangements of a same word. This property is given by the study of the kernel of the Klyachko invariant. For this part, we work in the general context of a connected cocommutative bialgebra $A$.

Theorem 6.0.6 The kernel of $\kappa_{n}$ restricted to $A_{n}$ is spanned by the elements of the form $a b-\omega^{|b|} b a \mathcal{C}_{|b|,|a|}((2,1))$.

Proof We directly use the proofs of Theorem 16, Lemma 14, and Corollary 15 given in [9]. The proof of Theorem 16 explicitly rests on the fact that primitive must be generating in $A$; hence the hypothesis on $A$.

First recall the principle of the proof. We examine a word $a_{1} a_{2} \ldots a_{p}$ with total degree $\left|a_{1}\right|+\left|a_{2}\right|+\cdots+\left|a_{p}\right|=n$, its image $\kappa_{n}\left(a_{1} a_{2} \ldots a_{p}\right)$, and the circular permutation $a_{p} a_{1} \ldots a_{p-1}$ and its image $\kappa_{n}\left(a_{p} a_{1} \ldots a_{p-1}\right)$. Both images are linear combinations of words obtained by permutations of $a_{1} a_{2} \ldots a_{p}$. Then, we focus our attention to such a word $w$. In the classical case one proves that the coefficient of $w$ in $\kappa_{n}\left(a_{p} a_{1} \ldots a_{p-1}\right)$ is equal to the coefficient of the same $w$ in $\kappa_{n}\left(a_{1} a_{2} \ldots a_{p}\right)$ multiplied by $\omega_{n}^{\left|a_{p}\right|}$.

Now we want to extend this classical case (the basis ring is a field $\mathbb{F}$ of characteristic 0 , possibly extended by primitive roots of unity) to the twisted case: the basis ring is the group ring $\mathbb{F}(\mathfrak{S})$. The coefficient of the word $w$ mentioned in the preceding paragraph results from two distinct processes. First, a linear combination in $\mathbb{F}$ of coefficients of the form $\omega_{n}^{\operatorname{maj}(C)}$ which appear in the definition of $\kappa_{n}$; notice that if, in the classical case, we calculate $p_{\left|a_{1}\right|} \star p_{\left|a_{2}\right|} \star \cdots \star p_{\left|a_{p}\right|}\left(a_{1} a_{2} \ldots a_{p}\right)$, where the $a_{1}, a_{2}, \ldots, a_{p}$ are primitive, we obtain a combination of words obtained by some permutations of the word $a_{1} a_{2} \ldots a_{p}$ with all coefficients equal to 1 . Secondly, the action of the group of permutations; it is generated by a repeated application of 
the following formula: $\delta\left(a_{1} a_{2}\right)=a_{1} a_{2} \otimes 1+a_{1} \otimes a_{2}+a_{2} \otimes a_{1} \mathcal{C}_{\left|a_{2}\right|,\left|a_{1}\right|}((2,1))+$

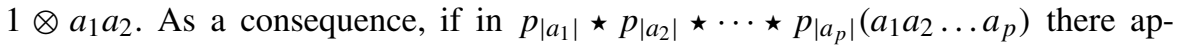
pears a word $a_{\sigma(1)} a_{\sigma(2)} \ldots a_{\sigma(p)}$ for some permutation $\sigma$, its coefficient in $\mathbb{F}(\mathfrak{S})$ is $\mathcal{C}_{\left|a_{\sigma(1)}\right|,\left|a_{\sigma(2)}\right|, \ldots,\left|a_{\sigma(p)}\right|}$.

So if we determine the coefficient of $w=a_{\sigma(1)} a_{\sigma(2)} \ldots a_{\sigma}(p)$ in $\kappa_{n}\left(a_{1} a_{2} \ldots a_{p}\right)$, we obtain the coefficient given in [9] multiplied by $\mathcal{C}_{\left|a_{\sigma(1)}\right|,\left|a_{\sigma(2)}\right|, \ldots,\left|a_{\sigma(p)}\right|}(\sigma)$.

Similarly if we determine the coefficient of $w=a_{\sigma(1)} a_{\sigma(2)} \ldots a_{\sigma(p)}$ in $\kappa_{n}\left(a_{p} a_{1} \ldots a_{p-1}\right)\left(-\omega_{n}^{\left|a_{p}\right|}\right) \mathcal{C}_{\left(\left|a_{p}\right|,\left|a_{1}\right|+\cdots+\left|a_{p-1}\right|\right)}((2,1))$, we obtain the coefficient given in [9] multiplied by $\mathcal{C}_{\left|a_{\sigma(1)}\right|,\left|a_{\sigma(2)}\right|, \ldots,\left|a_{\sigma(p) \mid}\right|}(\sigma)$, the same permutation as above (indeed a permutation is given by its image and in our case by the word $w$ ).

In conclusion, the proof of [9] still works in the twisted case.

We now go back to the case of $A=\mathcal{F}(X)$.

First we remark that the free twisted bialgebra on $X$ can be generated on $\mathbb{F}(\mathfrak{S})$ by the union of all $\{b(w)\}_{w \in L}$ and the nontrivial circular rearrangements of all $w$ in $L$ :

Lemma 6.0.7 Let $\left\langle L_{n}\right\rangle$ be the twisted module generated by $L_{n}$ in $\mathcal{F}(X), B_{n}$ its image by the linear extension of $b: L \rightarrow \mathcal{L}(X) \subset \mathcal{F}(X)$, and $K_{n}$ the kernel of the Klyachko idempotent $\kappa_{n}: \mathcal{F}(X)_{n} \rightarrow \mathcal{L}(X)_{n} \subset \mathcal{F}(X)_{n}$. Then there is an isomorphism between $\left\langle L_{n}\right\rangle \oplus K_{n}$ and $B_{n} \oplus K_{n}$.

Proof Look at the decomposition given by Theorem 4.2.2. Consider the basis of $B_{n}$ consisting of all $b(w), w \in L_{n}$, and order it lexicographically. Similarly we consider the basis of $\left\langle L_{n}\right\rangle$ consisting of all $w, w \in L_{n}$, again ordered lexicographically. Choose some basis for $K_{n}$. Then Theorem 4.2.2 implies that the matrix giving the basis of $B_{n} \oplus K_{n}$ in the basis of $\left\langle L_{n}\right\rangle \oplus K_{n}$ is a triangular matrix with 1 at each spot of the diagonal. This proves the lemma.

Remark Recalling that the Lyndon words are the representatives of all circular rearrangement classes of $W(X)$, we see that $\left\langle L_{n}\right\rangle \oplus K_{n}=\mathcal{F}(X)$.

We can now prove the following sequence of inclusions:

$$
\begin{aligned}
\mathcal{L}(X)_{n} \supseteq B_{n} & =\kappa_{n}\left(B_{n}\right) \\
& =\kappa_{n}\left(B_{n} \oplus K_{n}\right) \quad \text { by definition of } K_{n} \\
& =\kappa_{n}\left(\left\langle L_{n}\right\rangle \oplus K_{n}\right) \quad \text { by Lemma } 6.0 .7 \\
& =\kappa_{n}(\mathcal{F}(X)) \quad \text { by the above remark } \\
& =\mathcal{L}(X)_{n} \quad \text { by Milnor Moore, Theorem 3.3.3. }
\end{aligned}
$$

So we can state the following:

Theorem 6.0.8 As a twisted module, the free (twisted) Lie algebra is generated by the Lie elements associated to all Lyndon words.

Combining this with Theorem 4.2.2 we obtain our main result: 
Theorem 6.0.9 The set of Lie elements associated to all Lyndon words is minimal and generating for the free twisted Lie algebra.

To conclude with, we now return to [2] and specify:

(i) Each $b(w), w$ a prime Lyndon word, generates submodule isomorphic to $\mathbb{F}\left(\mathfrak{S}_{|w|}\right)$ in $\mathcal{L}(X)$.

(ii) Each $b(w), w=u^{p}, p>1$ and $u$ a prime Lyndon word, generates submodule isomorphic to $\mathbb{F}\left(\mathfrak{S}_{|w|}\right) / I_{p,|u|}$ in $\mathcal{L}(X)$, where $I_{p,|u|}$ is the annihilator of $\left(1-\gamma_{2}\right) \ldots\left(1-\gamma_{p}\right)$ (cf. Proposition 4.2.1).

Acknowledgements We are indebted to F. Patras for drawing our attention to Barratt's article on twisted Lie algebras and for pointing out that his assertion about their linear basis remains unproved. We are also grateful to the referee who incited us to clarify various notions of freeness for twisted objects.

\section{References}

1. Aubry, M.: Twisted Lie algebra and idempotent of Dynkin. Sémin. Lothar. Comb. 62 (to appear)

2. Barratt, M.G.: Twisted Lie algebras. Geometric applications of homotopy theory. In: Proc. Conf., Evanston, Ill., 1977, II. Lecture Notes in Math., vol. 658, pp. 9-15. Springer, Berlin (1978)

3. Fresse, B.: Koszul duality of operads and homology of partition posets. In: Homotopy Theory: Relations with Algebraic Geometry, Group Cohomology, and Algebraic $K$-theory. Contemp. Math., vol. 346, pp. 115-215. Am. Math. Soc., Providence (2004)

4. Garsia, A.M.: Combinatorics of the free Lie algebra and the symmetric group. In: Analysis, et Cetera, pp. 309-382. Academic Press, San Diego (1990)

5. Gelfand, I.M., Krob, D., Lascoux, A., Leclerc, B., Retakh, V.S., Thibon, J.-Y.: Noncommutative symmetric functions. Adv. Math. 112(2), 218-348 (1995)

6. Joyal, A.: Foncteurs analytiques et espèces de structures. In: Combinatoire Énumérative, Montréal, Qué., 1985/Québec, Que., 1985. Lecture Notes in Math., vol. 1234, pp. 126-159. Springer, Berlin (1986)

7. Livernet, M., Patras, F.: Lie theory for Hopf operads. J. Algebra 319(12), 4899-4920 (2008)

8. Markl, M., Shnider, S., Stasheff, J.: Operads in Algebra, Topology and Physics. Mathematical Surveys and Monographs, vol. 96. Am. Math. Soc., Providence (2002). x+349 pp.

9. Patras, F., Reutenauer, C.: On Dynkin and Klyachko idempotents in graded bialgebras. Special issue in memory of Rodica Simion. Adv. Appl. Math. 28(3-4), 560-579 (2002)

10. Patras, F., Reutenauer, C.: On descent algebras and twisted bialgebras. Mosc. Math. J. 4 311(1), 199216 (2004)

11. Reutenauer, C.: Free Lie Algebras. London Mathematical Society Monographs. New Series, vol. 7. Oxford Science Publications, The Clarendon Press, Oxford University Press, London (1993). xviii+269 pp.

12. Stover, C.R.: The equivalence of certain categories of twisted Lie and Hopf algebras over a commutative ring. J. Pure Appl. Algebra 86(3), 289-326 (1993) 